\title{
Tendencias metodológicas en la investigación académica sobre Comunicación
}

\author{
Tendencias metodológicas \\ en la investigación acadé- \\ mica sobre Comunicación \\ Carmen Caffarel Serra \\ uan Antonio Gaitán Moya \\ Carlos Lozano Ascencio \\ José Luis Piñuel Raigada \\ (Editores) \\ 100 \\ D 1 \\ 10 \\ $\circ>$ \\ 0 \\ 10

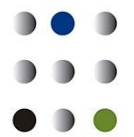 \\ Carmen Caffarel Serra, Juan Antonio \\ Gaitán Moya, Carlos Lozano Ascencio y \\ José Luis Piñuel Raigada \\ Editorial Comunicación Social, 2018 \\ 302 páginas \\ Reseña por Rosalba Mancinas-Chávez
}

Cualquier investigador en el área de comunicación en España habrá escuchado hablar de MapCom en los últimos años, algunos porque han sido contactados para entrevista, otros porque les han requerido los datos de su grupo de investigación y otros por haber atendido a las convocatorias de congreso realizadas por la Asociación Española de Investigadores en Comunicación (AE-IC), a través de su sección temática de Teoría y Métodos de Investigación en Comunicación (TMIC). El alcance de este proyecto de investigación ha sido bastante significativo, ha sentado unas bases que deberían continuarse porque no hay mejor manera de avanzar en investigación que conociendo bien lo que estamos haciendo unos y otros. Significa también que hacía falta realizar este esfuerzo de coordinación entre varios grupos en distintas regiones para trazar el mapa de qué y cómo estamos investigando en comunicación.

Tendencias metodológicas en la investigación académica sobre Comunicación forma parte de los resultados de MAPCOM (www.mapcom.es), una red de proyectos de investigación coordinados que comparten objetivos y metodología. Bajo la dirección de José Luis Piñuel, el equipo coordinador procede de la Universidad Complutense de Madrid (UCM); los otros tres equipos son de la Universidad del País Vasco (EHU), de la Universitat Jaume I (UJI) y de la Universidad de Málaga (UMA). Juntos forman un equipo de investigación potente que ha retratado de forma muy precisa el mapa de la investigación en comunicación en España, 
atendiendo al número de tesis y proyectos de investigación, como principales resultados, pero también haciendo un mapa de grupos de investigación disponible como herramienta en su página web.

La obra se presenta en dos partes. En la primera, los autores aportan tres capítulos que se corresponden con las tres fases del proyecto, pero más enfocados en los usos metodológicos de la investigación sobre las prácticas sociales de comunicación. En la segunda parte, el lector encontrará una selección de las ponencias presentadas en los congresos nacionales convocados por la sección TMIC de la AE-IC en 2015 y 2017. Se han seleccionado por su relevancia y por su capacidad para marcar tendencia en investigación en comunicación. Una docena de trabajos organizados en tres capítulos por la coherencia temática.

Es un libro denso, de consulta más que de lectura. Un hito importante en la "meta-investigación", investigar sobre la investigación en materia de comunicación.

Uno de los apartados más interesantes es el capítulo 2 que recoge el debate de los investigadores. De manera gráfica en las figuras 1 y 2 se representan las “... conceptualizaciones codificadas y sus relaciones en el discurso de los investigadores cuando se habla de Metodología en las sesiones plenarias", una serie de consideraciones que van desde el efecto ANECA hasta reconocer la falta de formación metodológica en comunicación y la falta de madurez en la disciplina. Una red de conceptos que nos llevan todos a la misma afirmación "faltan recursos para la investigación".

Obra de obligada consulta que servirá de base para futuras investigaciones, que ofrece muchas ideas para profundizar en ramas específicas de la comunicación, en zonas concretas y en momentos determinados. 